\title{
Características del SARS-CoV-2 y sus mecanismos de transmisión
}

\author{
SARS-CoV-2; Virus characteristics and transmission
}

\author{
Nancy Evelyn Aguilar Gómez,* Astrid Anaid Hernández Soto,` Cyntia Ibanes Gutiérrez ${ }^{\ddagger}$ \\ * Adscrito al Departamento de Infectología. \\ ‡ Residente de Infectología. \\ Instituto Nacional de Pediatría.
}

RESUMEN

\begin{abstract}
Desde los primeros casos descritos de infección por SARS-CoV-2 (COVID-19) en diciembre de 2019 en Wuhan (provincia de Hubei), China, la pandemia ha afectado a 216 países del mundo y ha causado un problema de salud pública. El conocimiento del virus y su capacidad para causar enfermedad ha sido el tema principal de investigaciones recientes; sin embargo, hay mucho que aún se desconoce. El conocimiento de las potenciales vías de transmisión del SARS-CoV-2 (respiratoria/gotas, indirecta, fecal-oral, vertical, sexual y ocular) son importantes en la práctica clínica, especialmente para la aplicación de medidas preventivas para mitigar la diseminación del SARS-CoV-2. A continuación, presentamos una revisión de la literatura de las características del virus, epidemiología y mecanismos de transmisión recientemente descritos.
\end{abstract}

Palabras clave: COVID-19, SARS-CoV-2, transmisión.

\section{DESCRIPCIÓN DEL VIRUS}

EI SARS-CoV-2 pertenece a la familia de los $\beta$-coronavirus, los cuales son virus de ácido ribonucleico (ARN) de cadena simple, polaridad positiva, envueltos, no segmentados, con genoma de 27 a $32 \mathrm{~kb}$ y tamaño de $80-160 \mathrm{~nm}$. Son los virus de ARN más grandes hasta ahora descubiertos y pertenecen al grupo IV de la clasificación de Baltimore. Se conocen hasta ahora cuatro géneros y se distinguen por el

Financiamiento: Ninguno.

Conflicto de intereses: Ninguno.

https://dx.doi.org/10.35366/95651

Rev Latin Infect Pediatr 2020; 33 (3): 143-148

\section{ABSTRACT}

Since the first described cases of SARS-CoV-2 infection (COVID19) in December 2019 in Wuhan, Hubei Province, China, the pandemic has affected 216 countries in the world and has caused a public health emergency. Understanding the virus and its ability to cause disease has been the main focus of current literature; however, there is much unknown. The knowledge of the potential routes of transmission of SARS-CoV-2 (respiratory/droplets, indirect, fecal-oral, vertical, sexual, and ocular) are important in clinical practice, especially in taking preventative measures to mitigate the spread of SARS-CoV-2. We present a review of the literature on the characteristics of the virus, epidemiology and transmission mechanisms recently described.

Keywords: COVID-19, SARS-CoV-2, transmission.

huésped al que infectan: $\alpha$-coronavirus: mamíferos, $\beta$-coronavirus (subdivididos en los grupos A-D): mamíferos, $\gamma$-coronavirus: aves, peces, y por último $\delta$-coronavirus: aves. ${ }^{2}$ En la actualidad, existen siete coronavirus que infectan al humano ${ }^{1}$ (Tabla 1).

Los tres coronavirus que afectan al humano y que tienen elevada patogenicidad son: síndrome Respiratorio Agudo Grave-1 (SARS-CoV-1), síndrome respiratorio de Oriente Medio (MERSCoV) y síndrome Respiratorio Agudo Grave-2 (SARS-CoV-2), los cuales se han caracterizado por asociarse a enfermedad grave. ${ }^{2}$ En la Tabla 2 se describe la fecha, origen, tipo de receptor, enfermedad causada, número de países afectados, total de casos confirmados, defunciones y número de reproducción básica (R0) hasta el día 8 de 
septiembre de 2020 de SARS-CoV-1, MERS-CoV y SARS-CoV-2. ${ }^{3}$

El genoma de SARS-CoV-2 tiene $96 \%$ de homología con un beta-coronavirus descrito en murciélagos y $91 \%$ con un beta-coronavirus del pangolín; de ahí que se considere como huésped definitivo (origen) e intermediario, respectivamente; 4,5 además de tener $80 \%$ de homología con el SARS-CoV- 1 y $55 \%$ con MERS-CoV. ${ }^{4,6}$ En China, se realizó un análisis filogenético de 103 cepas de SARS-CoV-2, en el que se identificaron dos haplotipos que difieren el uno del otro por dos polimorfismos de nucleótido único (SNP, por sus siglas en inglés), designados como tipo $L(70 \%)$ y tipo S (30\%). El tipo L predominó durante la etapa temprana de la epidemia en China, caracterizándose por hipovirulencia y mayor transmisión. ${ }^{5} \mathrm{Un}$ análisis filogenético más reciente publicado el 30 de marzo de 2020, de 160 genomas completos de SARS-CoV-2 de diferentes regiones del mundo, identificaron tres variantes o cepas: A, B y C por mutaciones en aminoácidos específicos, lo que permite caracterizar el desplazamiento geográfico y la evolución de cada una de éstas ${ }^{4}$ (Figura 1).

\section{INFECTIVIDAD}

El número de reproducción básica $(\mathrm{R} 0)$ para SARSCoV-2 es variable según el momento de la pandemia, la metodología empleada en los modelos para su cálculo y la aplicación de medidas preventivas efectivas. ${ }^{7}$ En la actualidad, el R0 se ha calculado por la Organización Mundial de la Salud (OMS) en 1.94 (IC 95\%: 1.4-2.5), similar al determinado en Wuhan de 2.2 (IC 95\%: 1.4-3.9). Sin embargo, se han reportado R0 de hasta 10 en pacientes conocidos como "súper transmisores». ${ }^{8}$
El incremento rápido de casos en ciertos contextos, por ejemplo, en el personal de salud, expone la posibilidad de que existan «súper transmisores», es decir, individuos con una capacidad de transmitir el virus mayor al percentil 95, generando un número de casos secundarios mucho mayor al esperado por los transmisores comunes. ${ }^{9}$ La «súper transmisión» responde a múltiples factores del virus (mutaciones puntuales), del hospedero (duración del contacto, comorbilidades), ambientales (recirculación de aire, densidad de población) y del comportamiento humano (visitas a múltiples médicos por parte de un mismo paciente, viajes y apego a lineamientos emitidos por las instancias de salud). ${ }^{10}$ Se ha documentado que los niños tienen una mayor prevalencia de enfermedad leve que pudiera pasar en ocasiones desapercibida, convirtiéndose en potenciales «súper transmisores», principalmente en las escuelas por ser sitios cerrados y concurridos. ${ }^{11}$ Nishiura y colaboradores demostraron una transmisión 18.7 veces mayor en ambientes cerrados, como gimnasios, restaurantes y hospitales, que en sitios bien ventilados. ${ }^{12}$ La tasa de ataque reportada para SARS-CoV-2 en contactos cercanos es de 35\% (IC 95\%: 27-44). ${ }^{12}$

\section{TRANSMISIÓN}

La transmisión de SARS-CoV-2 se ha descrito por mecanismos directos e indirectos:

Directos: SARS-CoV-2 puede transmitirse, como la mayoría de los virus respiratorios, mediante secreciones respiratorias, siendo éste el mecanismo principal de transmisión (persona a persona).

- Transmisión por gotas: tienen un tamaño > 5-10 $\mu \mathrm{m}$; se producen al hablar, toser, estornudar,

\begin{tabular}{|cccc|}
\hline \multicolumn{4}{c}{ Tabla 1: Descripción de los siete coronavirus descritos que infectan al humano. } \\
\hline Nombre & Fecha de descubrimiento & Género & Patogenicidad \\
\hline HCoV-229E & 1960 & Alfa & Baja \\
HCOV-OC43 & 1960 & Beta & Baja \\
SARS-CoV-1 & 2002 & Beta & Elevada \\
HCoV-NL63 & 2004 & Alfa & Baja \\
HCoV-HKU1 & 2005 & Beta-A & Baja \\
MERS-CoV & 2012 & Beta & Elevada \\
SARS-CoV-2 & 2020 & Beta & Elevada \\
\hline
\end{tabular}




\begin{tabular}{cccc}
\hline \multicolumn{2}{c}{ Tabla 2: Características de los coronavirus asociados con enfermedad } \\
grave: SARS-CoV-1, MERS-CoV y SARS-CoV-2.
\end{tabular}

cantar o respirar. Se desplazan aproximadamente un metro de distancia al hablar y hasta cuatro metros al toser o estornudar. ${ }^{13}$

- Transmisión por aerosoles: partículas $<5 \mu \mathrm{m}$ que quedan suspendidas en el aire ambiente siendo infectivas por al menos tres horas, con una mayor concentración en las fases iniciales de la enfermedad y durante la realización de procedimientos que generen aerosoles como intubación endotraqueal, broncoscopia y resucitación cardiopulmonar. ${ }^{13}$ Se desplazan aproximadamente de ocho a diez metros de distancia. ${ }^{14}$ Modelos experimentales han demostrado que en una conversación de 10 minutos, una persona infectada puede producir hasta 6,000 partículas de aerosoles. ${ }^{15,16}$

\section{Indirectos}

- La tercera vía de transmisión es por contacto, ya que el virus depositado en distintas superficies por las gotas o aerosoles producidos por un individuo infectado permanece viable por tiempo variable en función de las características del material. Así, el contacto con algún fómite y, posteriormente, con alguna mucosa (oral, nasal o conjuntival) puede ocasionar la infección.

Con base en experimentos realizados en virus similares tales como SARS-CoV, MERS-CoV y otros coronavirus, se ha determinado un tiempo promedio de viabilidad para SARS-CoV-2 en aluminio (de dos a ocho horas), cobre (cuatro horas), guantes quirúrgicos (ocho horas), plástico (72-96 horas), cartón (24-96 horas), acero inoxidable (48-72 horas), papel (cuatro a cinco días), vidrio y madera (cuatro días). ${ }^{15-17}$

\section{Otros mecanismos}

- Fecal-oral: este mecanismo de transmisión ocurre dada la capacidad de infección a las células del epitelio intestinal por la expresión del receptor de la enzima convertidora de angiotensina 2 (ACE2) y la proteasa TMPRSS2. ${ }^{13}$ Se ha reportado la excreción viral prolongada en materia fecal en pacientes asintomáticos. En un reporte de caso de un paciente con COVID-19 se detectó el virus en evacuaciones hasta por 42 días, mientras que el hisopado nasofaríngeo fue negativo. ${ }^{13}$ Además, la excreción viral prolongada se ha descrito en pacientes pediátricos, detectando el virus en evacuaciones posterior a 10 días de la remisión de los síntomas. ${ }^{13} \mathrm{~A}$ pesar de la documentación de excreción viral prolongada en evacuaciones, el potencial de que estas partículas sean infecciosas es cuestionable. ${ }^{19}$

- Vertical: aunque se ha estudiado la transmisión vertical de COVID-19, aún existe la necesidad de 
mayor evidencia para poder realizar conclusiones al respecto. En un estudio de Dong y colaboradores reportaron el caso de un recién nacido hijo de madre con COVID-19, se encontró elevada la IgM dos horas posteriores al nacimiento, pero el hisopado faríngeo para SARS-CoV-2 fue negativo. ${ }^{13}$ Contrario a lo anterior, en un estudio retrospectivo de nueve embarazadas con COVID-19 no se detectó el virus por RT-PCR en líquido amniótico, sangre de cordón umbilical, hisopado faríngeo o leche materna. ${ }^{14}$ Se necesitan más estudios para determinar si SARS-CoV-2 atraviesa la membrana placentaria. $^{14}$

- Sexual: en un estudio en el que examinaron 34 adultos recuperados de COVID-19 en muestras tomadas de semen, aproximadamente un mes posterior de la confirmación de la infección SARS-CoV-2, no se detectó el virus en las muestras de los pacientes. Se ha documentado positividad en etapas tempranas de la infección; sin embargo, se necesitan más estudios para determinar el rol de esta vía en la transmisión de SARS-CoV-2. ${ }^{13}$

- Ocular: esta vía de transmisión es recientemente explorada y los estudios que existen en la actualidad son limitados. Se ha documentado la expresión de receptores ACE2 en tejido de córnea y conjuntiva en menor proporción al compararse con el tejido pulmonar o corazón. ${ }^{20}$ CD147, una proteína transmembrana que está asociada a infección viral, promueve la invasión de SARS-CoV-2 a los tejidos y por estudios inmunohistoquímicos se ha detectado en el epitelio de la córnea, conjuntiva y retina. ${ }^{17}$ Existen pocos reportes de conjuntivitis por SARS-CoV-2 y los datos epidemiológicos reportan una incidencia de $0.8-4.8 \% .^{20}$ Se ha documentado RT-PCR positiva para SARS-CoV-2 en lágrimas y secreciones conjuntivales en pacientes con y sin conjuntivitis. ${ }^{20}$ Si bien esta vía de transmisión se ha demostrado en modelos experimentales, su incidencia es baja y es considerada una potencial vía de infección, principalmente en hospitales. ${ }^{20}$

- Sanguínea: por el momento no existe evidencia que sugiera la transmisión por transfusión de hemoderivados. ${ }^{21}$

Las estrategias tradicionales de control de infecciones de salud pública dependen en gran medida de la detección temprana de enfermedades para contener la propagación. Al inicio de la pandemia la medida de la intervención de los países incluía la detección de los pacientes sintomáticos, justificado por las similitudes con SARS-CoV-1 y MERS-CoV,
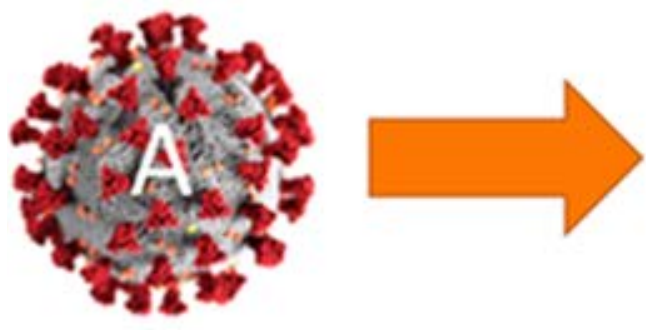

Tipo A

Coronavirus relacionado con el encontrado en murciélagos y pangolín

Considerado como el «origen de la epidemia»

Asociados a brotes en Wuhan, América y Australia
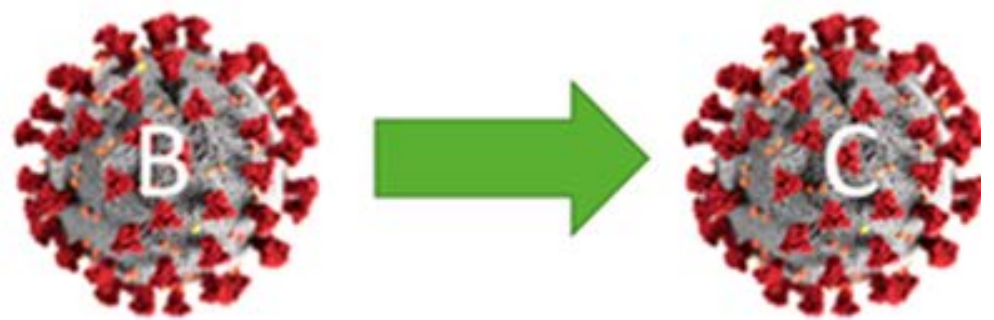

\begin{tabular}{|c|c|}
\hline $\begin{array}{c}\text { Variación del corona- } \\
\text { virus más común en } \\
\text { Wuhan }\end{array}$ \\
\hline Derivado del A por dos \\
mutaciones: T8782C y \\
C28144T \\
(leucina $\times$ serina) \\
\hline Mutó lentamente en \\
China y rápidamente \\
fuera de China (México) \\
\hline
\end{tabular}

Tipo C

Es la descendiente del linaje B

Derivado del B por unas mutaciones:

G26144T (glicina $\times$ valina)

Distribución en Europa vía Singapur

Datos adaptados de: Forster P, Forster L, Renfrew C et al. Phylogenetic network analysis of SARS-CoV-2 genomes. PNAS. 2020; 117 (17): $9241-9243$.

Figura 1: Tres cepas de coronavirus identificadas, mutaciones específicas y distribución geográfica. 
Rev Latin Infect Pediatr 2020; 33 (3): 143-148

con el desconocimiento de otros mecanismos de contagio. Hoy en día, se sabe que los individuos asintomáticos y presintomáticos son importantes en la transmisión del virus.

Asintomáticos: diversos autores han descrito la existencia de portadores asintomáticos, capaces de transmitir el virus e incluso de desarrollar lesión pulmonar demostrada por imagen a pesar de no presentar ninguna manifestación clínica. ${ }^{15} \mathrm{La}$ proporción de portadores asintomáticos se ha calculado hasta en $17.9 \%$, como fue el caso del crucero Diamond Princess. ${ }^{16}$

Arons y colaboradores estudiaron durante un brote, en el estado de Washington, a pacientes del centro hospitalario, y que fueron voluntarios para realizarse detección con RT-PCR en hisopados nasofaríngeos. Este estudio reportó que 27 de 48 pacientes positivos fueron asintomáticos y fueron potenciales transmisores de la infección. ${ }^{19}$

Transmisión presintomática: de manera similar, es posible la transmisión presintomática, es decir, durante el periodo de incubación, este es un factor clave para la transmisión de SARSCoV-2 dada la elevada excreción viral en el tracto respiratorio superior, incluso en pacientes presintomáticos. ${ }^{17}$

En estudios realizados en China, se documentó que el contagio puede ocurrir en la fase presintomática, uno a tres días previos al inicio de los síntomas. ${ }^{20}$ Así, se han reportado contagios en familias durante el periodo de incubación de un caso índice. ${ }^{19} \mathrm{La}$ prevalencia de pacientes con RT-PCR positiva para SARS-CoV-2 en fase presintomática varía de 30 a $60 \%$. Por ejemplo, Arons y colegas ${ }^{19}$ encontraron en su población de estudio que $43 \%$ (10/23) de pacientes con RT-PCR positiva para SARS-CoV-2 inició con síntomas tras una semana de la toma de muestra, conformando, por lo tanto, el grupo de los pacientes positivos en fase presintomática. ${ }^{19,22}$ En Singapur, la transmisión durante el periodo de incubación se estimó en 10 de 157 casos (6.4\%) y la exposición ocurrió aproximadamente uno a tres días antes del desarrollo de síntomas. ${ }^{21}$

\section{CONCLUSIONES}

La pandemia de COVID-19 muestra la importancia de realizar un adecuado control de la infección mediante la identificación de las diferentes vías de transmisión del virus. A pesar de que se han descrito la vía respiratoria y el contacto, existe limitada información de otras vías de transmisión como vertical, ocular, sexual y sanguínea. Estos hallazgos enfatizan la importancia de las medidas de control que pueden impactar en la práctica diaria, desde las precauciones que deben realizarse en el público en general para prevenir la transmisión presintomática hasta las medidas de precauciones específicas a nivel hospitalario.

\section{REFERENCIAS}

1. Bennett JE, Dolin R, Blaser MJ, editors. Principles and practice of infectious diseases. 9th ed. Philadelphia: Churchill Livingstone, 2020.

2. Cui J, Li F, Shi ZL. Origin and evolution of pathogenic coronaviruses. Nat Rev Microbiol. 2019; 17 (3): 181-192.

3. Coronavirus disease (COVID-19) outbreak situation. (2020). Recovered September 8, 2020, World and Health Organization website: https://www.who.int/emergencies/ diseases/novel-coronavirus-2019.

4. Forster $\mathrm{P}$, Forster $\mathrm{L}$, Renfrew $\mathrm{C}$ et al. Phylogenetic network analysis of SARS-CoV-2 genomes. PNAS. 2020; 117 (17): 9241-9243.

5. Tang $\mathrm{X}, \mathrm{Wu} \mathrm{C}$, Li $\mathrm{X}$ et al. On the origin and continuing evolution of SARS-CoV-2. National Science Review. 2020; 7 (6): 1012-1023.

6. Liu Y, Eggo RM, Kucharski AJ. Secondary attack rate and superspreading events for SARS-CoV-2. Lancet. 2020; 395 (10227): e47.

7. Kolifarhood G, Aghaali M, Mozafar SH et al. Epidemiological and clinical aspects of COVID-19; a narrative review. Arch Acad Emerg Med. 2020; 8 (1): e41.

8. Tang $\mathrm{B}$, Wang $\mathrm{X}$, Li $\mathrm{Q}$ et al. Estimation of the transmission risk of the 2019-nCoV and its implication for public health interventions. J Clin Med. 2020; 9 (2): 462.

9. Gralinski LE, Menachery VD. Return of the coronavirus: 2019-nCoV. Viruses. 2020; 12 (2): 135.

10. Wong G, Liu W, Liu Y et al. MERS, SARS, and Ebola: the role of super-spreaders in infectious disease. Cell Host Microbe. 2015; 18 (4): 398-401.

11. Liu Y, Eggo RM, Kucharski AJ. Secondary attack rate and superspreading events for SARS-CoV-2. Lancet. 2020; 395 (10227): e47.

12. Nishiura $H$, Oshitani $H$, Kobayashi $T$ et al. Closed environments facilitate secondary transmission of coronavirus disease 2019 (COVID-19). 2020. doi: https:// doi.org/10.1101/2020.02.28.20029272.

13. Patel K, Vunnam S, Patel P et al. Transmission of SARSCoV-2: an update of current literature. Eur J Clin Microbiol Infect Dis. 2020: 1-7. Available in: https://doi.org/10.1007/ s10096-020-03961-1.

14. Asadi S, Bouvier N, Wexler A et al. The coronavirus pandemic and aerosols: does COVID-19 transmit via expiratory particles? American Association for Aerosol Research. 2020; 635-638.

15. Van Doremalen N, Bushmaker T, Morris DH et al. Aerosol and surface stability of SARS-CoV-2 as compared with SARS-CoV-1. N Engl J Med. 2020; 382 (16): 1564-1567.

16. Blocken B, Malizia F, van Druenen T, Marchal T. Towards aerodynamically equivalent COVID-19 $1.5 \mathrm{~m} 180$ social distancing for walking and running. Urban physics, wind engineering \& sports aerodynamics, 2020. 
17. Van Doremalen N, Bushmaker T, Morris DH et al. Aerosol and surface stability of SARS-CoV-2 as compared with SARS-CoV-1. N Engl J Med. 2020; 382 (16): 1564-1567.

18. Shereen M, Khan S, Kazmi A et al. COVID-19 infection: origin, transmission, and characteristics of human coronaviruses. Journal of Advanced Research. 2020; 24: 91-98.

19. Arons MM, Hatfield KM, Reddy SC et al. Presymptomatic SARS-CoV-2 infections and transmission in a skilled nursing facility. N Engl J Med. doi: 10.1056/NEJMoa2008457.

20. Chen X, Yu H, Mei T. SARS-CoV-2 on the ocular surface: is it a truly novel transmission route? $\mathrm{Br} \mathrm{J}$ Ophthalmol. 2020; 0: 1-6.
21. Chang L, Yan Y, Wang L. Coronavirus disease 2019 coronaviruses and blood safety. [published online ahead of print, 2020 Feb 21]. Transfus Med Rev. 2020; doi: 10.1016/j. tmrv.2020.02.003.

22. Qian G, Yang N, Yan MA et al. COVID-19: transmission within a family cluster by presymptomatic carriers in China. Clin Infect Dis. 2020; 71 (15): 861-862.

Correspondencia:

Nancy Evelyn Aguilar Gómez

E-mail: nancyevelyn_061004@hotmail.com 